SHS Web of Conferences 21, 03008 (2015)

DOI: $10.1051 /$ shsconf $/ 20152103008$

(C) Owned by the authors, published by EDP Sciences, 2015

\title{
Mise en scène théâtrale de la réflexion bioéthique autour des soins palliatifs et de la fin de vie par des élèves de première scientifique
}

\author{
Géraldine Dargent \\ Centre International Valbonne, 190 rue Frédéric Mistral, 06560 Valbonne Sophia Antipolis, France
}

\begin{abstract}
Résumé. L'atelier de bioéthique du Centre International de Valbonne concerne douze élèves de $1^{\text {ère }}$ scientifique et se déroule sur le temps péri- scolaire. Ce groupe d'élèves, encadré par une équipe pédagogique et des intervenants du domaine de la santé et du théâtre a réfléchi sur le thème des soins palliatifs et de la fin de vie. Cette réflexion a été mise en scène par les élèves eux-mêmes, sous la forme d'une pièce de théâtre pour être présentée à la Journée de Bioéthique Jeunes à Aix en Provence, le 24 mars 2015. L'objectif de cet atelier a été de faire réfléchir les élèves sur leur position au sujet de la fin de vie et de susciter le débat et les échanges. Nous montrons dans cet article comment un atelier permet aux élèves d'exprimer et de faire évoluer leurs points de vue à travers une pièce de théâtre sur le thème des soins palliatifs et de la fin de vie.
\end{abstract}

\begin{abstract}
The bio-ethics club of CIV has twelve 11th level science studentsand takes place in school time. This group of students, helped by a pedagogical team and visitors from the fields of health and theatre, has thought about the theme of pallative care, end of life care. This reflection has been acted out by the students themselves for a piece of theatre to be presented at the Bio-ethic Young Person's Day at Aix en Provence on $24^{\text {th }}$ March 2015. The objectif of this worl has been to make the students their opinion on the subject of the end of life and to provoke debate and exchange. We are showing in this article how a club allows students to express themselves and develop their points of view through a piece of theatre on the theme of pallative care and the end of life.
\end{abstract}

L'année 2015 a été marquée, au niveau parlementaire, par un débat majeur autour de la fin de vie. Le 10 mars 2015, l'Assemblée Nationale a examiné la proposition de loi en faveur des malades et des personnes en fin de vie présentée par Alain Claeys et Jean Leonetti. Dans ce contexte, et comme le thème général de la journée Bioéthique Jeunes était "L'état de conscience », j'ai décidé de faire travailler les élèves de l'atelier de Bioéthique du lycée sur les soins palliatifs et l'accompagnement des personnes en fin de vie. Par ailleurs, ce thème semblait pertinent pour des élèves dont une majorité se destinait à des études de médecine; de plus, ce thème permettait de réfléchir à des questions socialement vives tout en développant l'esprit critique et l'argumentaire. L'objectif était de permettre aux élèves de construire une situation qui suscite le débat et les échanges. 


\section{Les étapes du projet}

\subsection{L’atelier bioéthique : une approche pluridisciplinaire Sciences, Art et Philosophie}

La journée «Bioéthique Jeunes», encadrée par des membres du Comité Consultatif National d'Ethique (CCNE) s'est tenue le mardi 24 mars 2015 dans la région d'Aix en Provence ; elle a réuni un ensemble d'établissements et de personnels du CCNE pour présenter et débattre de questions de bioéthique. L'année dernière, des élèves de Première du Centre International de Valbonne (CIV) avaient déjà participé à cette manifestation.

Nous avons souhaité renouveler ce projet en le plaçant- selon les directives du CCNE-dans un cadre pluridisciplinaire. Une équipe de trois enseignants s'est engagée dans ce projet: Mme Denépoux en Philosophie, Mme Aubanel en Arts Plastiques et Mme Dargent en Sciences de la Vie. Le point de départ de cette réflexion a été la loi Leonetti (2005), le rapport du CCNE du 21 octobre 2014 sur la fin de vie et les ouvrages de Marie De Hennezel et de David Servan Schreiber.

Le sujet retenu a été celui des Soins palliatifs et de la fin de vie rattaché au thème général sur la Conscience pour la Journée de Bioéthique Jeunes de cette année. Dès le départ, le projet a été de réaliser une pièce de théâtre et ceci pour plusieurs raisons : d'abord, la présentation se faisant devant un public d'élèves, la forme théâtrale me semblait plus ludique donc plus efficace surtout pour un sujet difficile à traiter comme la fin de vie. Ensuite, le jeu de rôle a permis à chaque élève de s'exprimer à travers un personnage donc sans s'exposer directement; enfin la perspective de créer puis de jouer sur scène a été très motivante pour les élèves. Comme pour tout projet, le traitement du sujet s'est fait de manière pluridisciplinaire ; la contribution de la Philosophie a consisté à réfléchir à quelles conditions un acte peut être considéré comme moral (énoncés de l'impératif catégorique de Kant); elle a permis également de discuter des notions de conscience, de liberté individuelle et d'éthique (énoncé de l'acte prudent d'Aristote). La contribution des Arts Plastiques a porté sur la réalisation d'une structure représentant le patient. La structure a été construite en plexiglas : la transparence permettait d'évoquer la fragilité du corps tandis que la rigidité évoquait la maladie et la souffrance. Une lampe multicolore télécommandée a été fixée sur la structure pour évoquer les états de conscience et les états émotionnels du patient (couleurs froides/ chaudes selon les états stress/apaisement du patient). Comme enseignante de SVT, j'ai pu apporter ma contribution sur les bases physiologiques de la douleur et le mécanisme de la sédation.

Je prévoyais au départ de parler de l'atelier à toutes les classes de Première. J'ai commencé à proposer l'atelier à la classe de Première $S$ dont j'étais le professeur principal ; 12 élèves volontaires se sont inscrits; l'effectif me semblant suffisant je n'ai pas proposé l'atelier à d'autres classes. L'atelier s'est déroulé à raison d'une heure par semaine de janvier à mars 2015 avec deux séances préparatoires l'une en novembre et l'autre en décembre 2014.

\subsection{Première séance : rencontre avec Mme Deligny, infirmière en soins palliatifs à domicile - 20 novembre 2014}

Cette rencontre a marqué le coup d'envoi de l'atelier de bioéthique. La présentation et le témoignage de Mme Deligny sur sa pratique professionnelle ont captivé les élèves qui ont volontiers échangé sur ces questions difficiles. Plusieurs questions ont porté sur le rôle des soins palliatifs :

"Avez-vous déjà sauvé des patients dans votre service de soins palliatifs? " " A quoi servent les soins palliatifs puisque le patient est condamné? "

D'autres interventions d'élèves ont abordé plus ou moins directement la question de l'euthanasie :

" Que faites-vous si la personne réclame de mourir? » 
«Est-ce que vous ne risquez pas de provoquer la mort du patient en lui injectant une dose de morphine trop importante?"

" Chacun doit pouvoir choisir sa propre mort. Pourquoi l'euthanasie reste-t-elle interdite en France?"

Lors de cette rencontre, les élèves ont exprimé leur position personnelle par rapport à la fin de vie. Plusieurs d'entre eux se sont prononcés, dans le cadre de la fin de vie, en faveur du " faire mourir », c'est-à-dire pour la pratique de l'euthanasie dans une situation incurable. Pour la majorité, au début de l'entretien, la notion de soins palliatifs n'était pas compréhensible; c'était un paradoxe de prodiguer des soins à une personne qui de toute façon est condamnée. A la suite de cette rencontre, les réponses de l'infirmière ont permis aux élèves de comprendre le rôle des soins palliatifs.
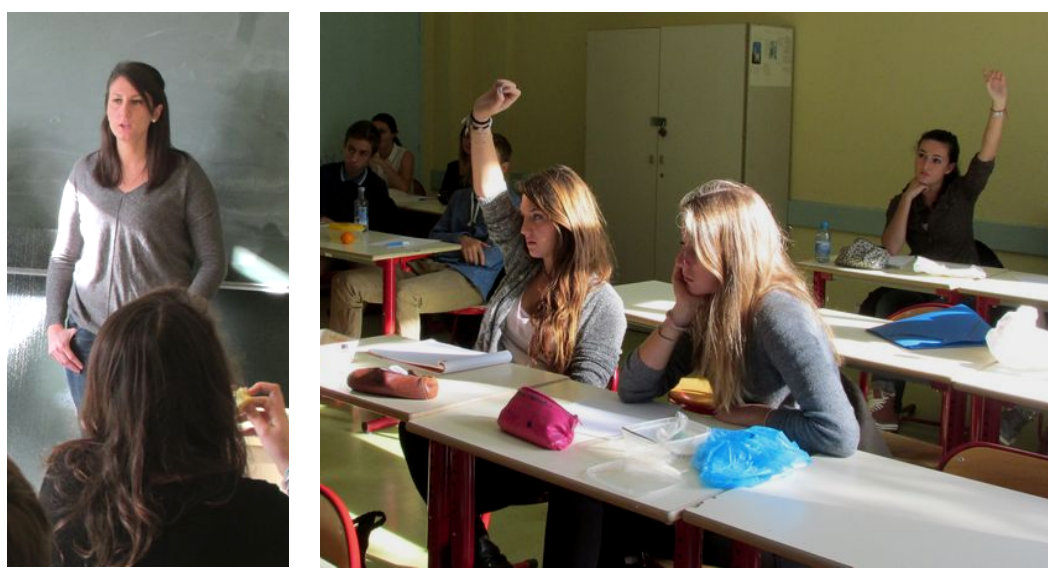

Photo 1. Mme Deligny, infirmière en soins palliatifs répond aux questions des élèves de l'atelier et fait part de son expérience professionnelle.

\subsection{Rencontre avec M. Lecomte et Mme Estival autour des soins palliatifs -18 décembre 2014}

Les élèves de l'atelier de bioéthique ont reçu M. Lecomte, comédien et metteur en scène et Mme Estival, coordonnatrice de la journée de Bioéthique Jeunes. A cette occasion les élèves ont présenté une sélection de scènes pour une représentation théâtrale d'une situation de soins palliatifs. M. Lecomte et Mme Estival ont pu réagir à cette présentation et y apporter leur contribution respective. Les échanges entre élèves, intervenants et professeurs ont permis de faire avancer la réflexion pour préparer les élèves à l'écriture de leur représentation.

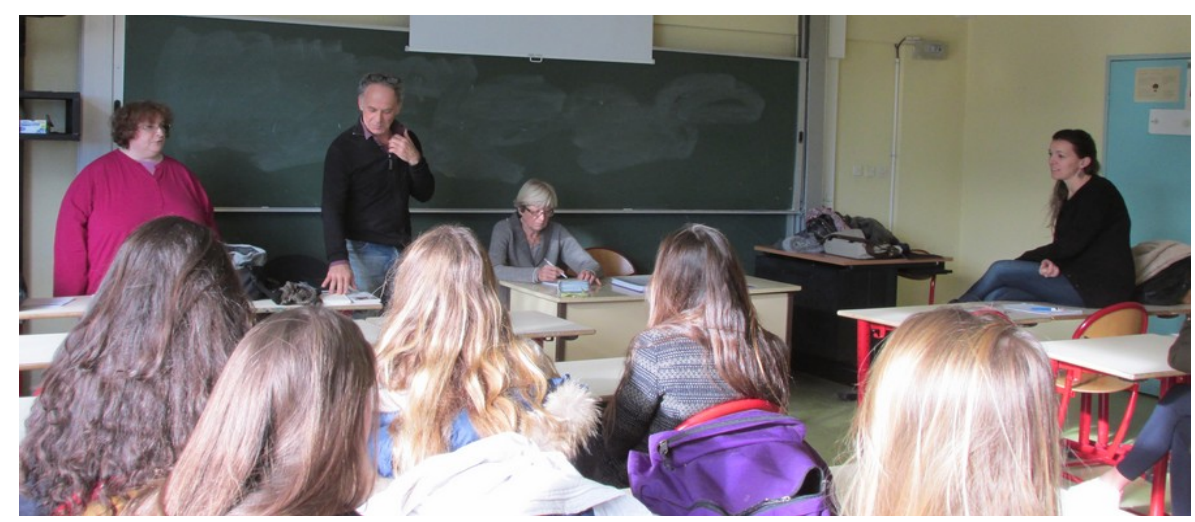

Photo 2. M. Lecomte et Mme Estival devant les élèves et les professeurs de l'atelier de bioéthique. 


\subsection{Sélection et écriture des scènes}

Les élèves ont constitué des groupes, chacun chargé de l'écriture d'une scène de la pièce. L'écriture s'est faite à la maison par les élèves en charge de la scène et suite aux échanges et débats durant les séances d'atelier. La pièce, d'une durée de vingt minutes, s'est composée de quatre scènes, chacune abordant un moment de l'histoire d'un adolescent malade, Alexandre, atteint d'un ostéosarcome.

Scène 1: Acharnement thérapeutique

$\underline{\text { Scène 2: }}$ Transfert du patient dans une unité de soins palliatifs

Scène 3: Aggravation de l'état du patient

$\underline{\text { Scène } 4}$ : Échanges dans l'entourage du patient pour décider que faire pour accompagner la toute fin de vie du patient. Confrontation d'opinions.

Dans le groupe, plusieurs élèves étaient en faveur de l'euthanasie et voulaient présenter cette prise de position dans une dernière scène ; la difficulté a été de faire comprendre à ces élèves que l'objectif de l'atelier n'était pas de présenter une position personnelle mais de créer une situation pour susciter le débat et le questionnement.

\section{5 Séance d'entraînement au CIV : présentation devant un public d'élèves de Terminale}

Les élèves de l'atelier de Bioéthique ont joué sur scène leur propre pièce de théâtre sur le thème de l'accompagnement de fin de vie. Cette représentation s'est faite devant un public d'élèves ayant réfléchi en classe à la question. Elle a été suivie d'un échange entre les acteurs et le public. Ces échanges ont porté sur la législation et sur les points de vue personnels des élèves. Cette rencontre a permis de préciser la mise en scène de la scène finale et a permis aux acteurs de développer une argumentation dans le cadre légal et en accord avec leurs diverses sensibilités.

Le fait qu'il s'agisse d'un public de terminale a permis d'aborder des points de vue avec des références philosophiques.

\subsection{Journée Nationale de Bioéthique Jeunes- Aix-en-Provence- Mardi 24 mars 2015}

Les élèves de l'atelier de Bioéthique accompagnés de l'ensemble de la classe ont participé mardi 24 mars à la Journée Nationale de Bioéthique Jeunes à Aix-en-Provence. Leur présentation s'est déroulée devant plusieurs établissements et un jury composé de représentants du Comité Consultatifs National d'Ethique : Mme Huet Véronique, Présidente des Ateliers de Réflexion Ethique Jeunes, Mme Estival Sylvette, Coordonnatrice de la Journée de Bioéthique Jeunes, et par des professionnels de santé :Mme MALZaC Perrine- Généticienne, Coordonnatrice de l'Espace éthique méditerranéen et M. BAGHDADI Houtin- Urgentiste réanimateur, Président du Comité de réflexion éthique des hôpitaux du Pays d'Aix et du Pertuis. La représentation a été suivie d'échanges avec le public et le jury.

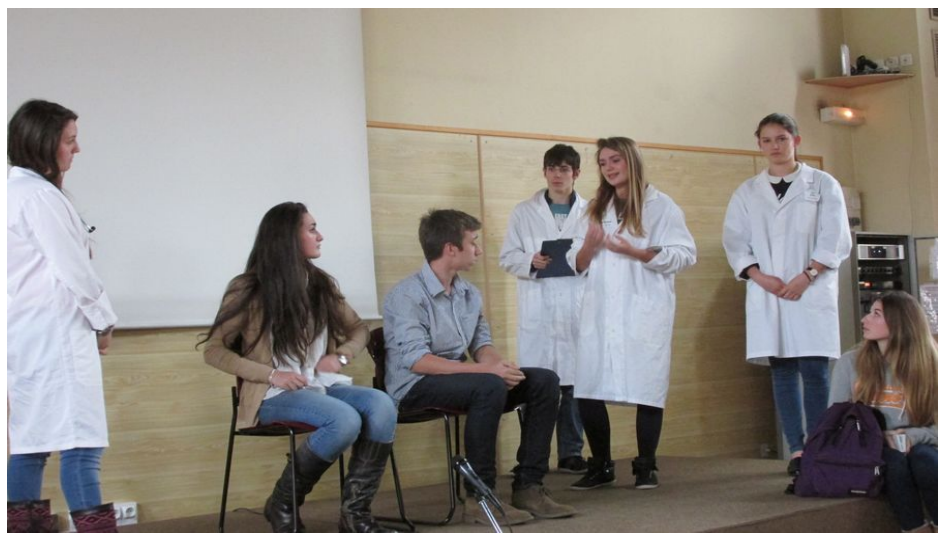

Photo 3 : Représentation sur scène des élèves de l'atelier de Bioéthique. 


\section{Comment un atelier de réflexion Bioéthique fait-il évoluer les représentations d'élèves de 1ère $S$ sur le thème de la fin de vie ? \\ 2.1 Questionnaire proposé à l'ensemble de la classe après l'action de l'atelier}

Le questionnaire ci-dessous a été proposé à l'ensemble de la classe de Première $\mathrm{S}$, en distinguant les élèves ayant suivi l'atelier de ceux ne l'ayant pas suivi ; l'objectif de cette démarche était d'évaluer l'impact de l'atelier sur les représentations des élèves sur le thème des soins palliatifs et de la fin de vie.

Cocher la bonne proposition

N'a pas participé à l'atelier de Bioéthique cette année A pas participé à l'atelier de Bioéthique cette année.

\section{Question 1 :}

D'après vous, qu'est-ce que « les soins palliatifs »?

\section{Question 2 :}

Parmi les pratiques d'accompagnement de fin de vie, laquelle (lesquelles) est (sont) légale(s) en

France (cocher la ou les bonne(s) réponse(s)) :

- la sédation profonde :

- l'acharnement thérapeutique :

- le suicide assisté :

- l'euthanasie :

Question 3 : Cocher la bonne réponse:

Selon vous, réfléchir à la fin de vie, c'est :

- utile à votre âge :

- $\quad$ sans intérêt à votre âge :

Justifiez :

Question 4 : Cocher la bonne réponse :

Avant cette année, vous aviez déjà réfléchi à la question de la fin de vie car :

- un cas s'est présenté dans votre famille ou votre entourage :

- $\quad$ vous en avez entendu parler dans les médias :

- $\quad$ vous avez vu un film/ lu un livre sur le sujet :

- $\quad$ autre raison (précisez laquelle) :

Avant cette année, vous n'aviez jamais réfléchi à la question de la fin de vie.

\section{Question 5 :}

Avez-vous actuellement une opinion personnelle sur la fin de vie?

\section{Question 6 :}

Votre opinion a -t-elle toujours été la même ou bien a t-elle évolué, changé ? Si oui, pourquoi ?

\section{Question 7 :}

Que pourriez-vous dire aux personnes qui n'ont pas la même opinion que vous sur la fin de vie?

\subsection{Extraits de réponses d'élèves au questionnaire}

À la question: Qu'est-ce que les soins palliatifs?

Tous les élèves arrivent à donner une définition correcte : "soins apportés par une équipe médicale pour accompagner une personne en fin de vie le but est de diminuer au maximum la douleur pour donner la fin la plus agréable au patient ». 


\section{À la question: Selon vous, réfléchir à la fin de vie est utile ou sans intérêt à votre âge?}

Une forte proportion d'élèves (24/30) considère que la réflexion sur la fin de vie est utile. "Nous ne sommes pas moins exposés aux accidents ou aux maladies que les personnes âgées." "

"Ce n'est pas parce qu'on est jeune qu'on ne doit pas être concerné par la fin de vie. "

Plusieurs élèves pensent que leur avis risque de changer aussi au cours du temps et qu'il est important d'en parler pour se faire une opinion. Cependant, même s'ils considèrent la réflexion utile, aucun n'est prêt à rédiger ses directives anticipées comme ils l'ont exprimé lors des échanges avec les membres du jury au cours de la journée Nationale de Bioéthique.

\section{À la question: Avez-vous votre propre opinion sur la fin de vie et a-t-elle évolué au cours de l'année?}

12/30 : en faveur de l'euthanasie active dans les extrêmes:

"Si je souffrais d'une maladie incurable, je préfèrerais mourir rapidement plutôt que de vivre alité. »

2/30 : contre l'euthanasie car «nous n'avons pas le droit de vie ou de mort sur qui que ce soit. »

1/30: pour l'euthanasie passive:

"Si un individu n'est plus dans la capacité de vivre sans machines et sans pouvoir bouger, il serait préférable de le laisser partir."

Les 15 autres élèves ne prennent pas partie sur la fin de vie. Le suivi de l'atelier les a aidé à préciser leur opinion mais il ne l'a pas modifiée:

"Mon opinion a évolué car avant je ne me posais pas assez la question; grâce à l'atelier, j'ai pu me faire une opinion. "

"Je n'ai pas changé d'avis, mais plus je découvre de choses sur le sujet, plus je me rends compte qu'il est difficile d'encadrer correctement une pratique comme l'euthanasie. »

Un changement radical d'opinion se fait quand la personne vit réellement la situation: " Mon opinion a changé car j'ai vu des personnes dans mon entourage en fin de vie. »

\section{Bilan}

L'analyse des réponses au questionnaire montre que le suivi de l'atelier de Bioéthique a permis aux élèves d'acquérir des connaissances plus complètes sur ce que sont les soins palliatifs en France et à l'étranger; les élèves ont également pris conscience de l'importance de l'accompagnement de la fin de vie. Plus particulièrement, les élèves ont réfléchi à la loi Leonetti de 2005 et à sa révision en mars 2015 (rapport Claeys-Leonetti) qui encadre la fin de vie en France.

De plus, avec l'atelier, les élèves ont affiné leur réflexion; ils ont appris à identifier les conflits de valeurs qui se jouent dans ces situations de soins palliatifs, à distinguer les cas particuliers du cas général, à réfléchir à ce qui est moral et ce qui ne l'est pas avec les références philosophiques de Kant et d'Aristote. Grâce à cette réflexion et à la construction de la mise en scène, les élèves ont pu se forger une opinion basée sur des données scientifiques et médicales plutôt que sur de simples impressions personnelles ou influences familiales. Plusieurs élèves qui avaient une position catégorique en faveur de l'euthanasie ont accepté d'ouvrir le débat et de rediscuter leur position. Les témoignages de l'infirmière en soins palliatifs sur des situations concrètes ont interpellé les élèves qui ont pris conscience que chaque situation est particulière et qu'il n'existe pas une réponse unique, chaque cas devant être rediscuté.

Par ailleurs, même si les élèves insistent sur l'importance des directives anticipées, ils ne sont pas prêts à les rédiger. Il reste toujours un décalage entre les intentions et les actions.

L'atelier a permis également d'acquérir des savoir-faire : savoir argumenter et débattre mais aussi savoir s'exprimer devant un public, savoir travailler en groupe comme à l'occasion de l'écriture des scènes. Enfin, l'atelier a permis de créer une véritable cohésion et dynamique au sein du groupe 
d'élèves ayant suivi l'atelier. A l'initiative des élèves eux-mêmes, une vidéo de la représentation sera réalisée en septembre prochain.

Par rapport aux élèves qui n'ont pas suivi l'atelier, les élèves de l'atelier ont davantage de connaissances sur l'organisation et la légalisation des soins palliatifs. Les échanges et la lecture de textes ont permis aux élèves de l'atelier d'acquérir ces connaissances. Les élèves qui n'ont pas suivi l'atelier ont également une opinion qui n'évolue pas sur les soins palliatifs. Au contraire pour les élèves ayant suivi l'atelier, leur opinion a évolué au contact des rencontres avec les experts l'infirmière en soins palliatifs, la généticienne lors de la rencontre à Aix-en-Provence- des débats et de l'écriture des scènes. Des élèves de l'atelier qui étaient au début farouchement en faveur de l'euthanasie tout en sachant que c'est illégal en France ont eu à la fin de l'atelier des positions plus modérées, réfléchies prenant en compte le cadre légal.

Les différentes approches disciplinaires ont permis de répondre plus largement à la diversité de sensibilité des élèves et d'avoir des éclairages différents sur le sujet ; l'apport de la philosophie a permis aux élèves d'apprendre à raisonner différemment. Pour l'enseignant meneur du projet, le travail pluridisciplinaire permet d'avoir des relais auprès de ses collègues et de redéfinir en permanence avec eux le cadre dans lequel on souhaite faire travailler les élèves. Cette expérience a été très positive et très riche pour chaque personne de l'atelier aussi bien sur le plan cognitif que sur le plan humain. Il aurait été intéressant cependant que l'ensemble de la classe s'associe au projet mais cela aurait nécessité que plus de temps soit consacré à l'atelier.

\section{Références}

1. M. Huber, Apprendre en projet (Chronique Sociale, Lyon, 2005).

2. M. De Hennezel, La mort intime: ceux qui vont mourir nous apprennent à vivre (Robert Laffont, Pocket, Paris, 2006).

3. Rapport du CCNE sur le débat public concernant la fin de vie (21 octobre 2014).

4. J. Ricot, Ethique du soin ultime (Presses de l'EHESP, Rennes, 2010).

5. D. Servan-Schreiber, On peut se dire au revoir plusieurs fois (Robert Laffont, Paris, 2011). 
